\title{
COMMENT
}

\section{In praise of model organisms}

\author{
Gregory A Petsko*
}

One of the many things I have against former Republican Vice-Presidential candidate Sarah Palin is not her lack of intelligence - she may in fact be intelligent, although she doesn't act like it (but then, I know a number of intelligent people who behave that way). But if she isn't very smart, that's not her fault. We all have to play the cards we're dealt. What I do have against Sarah Palin is her ignorance, because she has had ample opportunity to do something about that, and hasn't bothered even to try. In my view, she is not just ignorant; she's willfully ignorant, which I find inexcusable.

Anything that might disturb her comfortable, distorted view of reality is not just ignored - it's often ridiculed. In October 2008, in a speech she gave on autism and disabilities, she had this to say:

"This is a matter of how we prioritize the money that we spend. We've got a three trillion dollar budget, and Congress spends some 18 billion dollars a year on earmarks for political pet projects. That's more than the shortfall to fully fund the IDEA [Individuals with Disabilities Education Act]. And where does a lot of that earmark money end up? It goes to projects having little or nothing to do with the public good - things like fruit fly research in Paris, France... I kid you not."

The quote doesn't do justice to the mocking inflection of her voice as she says 'fruit fly research', but there are ample recordings floating around the Web if you care to hear it.

Of course, one retort might be that the average fruit fly may well be better informed than Sarah Palin, but ad feminem attacks shouldn't win any argument, and besides, some targets are just too easy. No, the real problem with her remark isn't that it comes from a clueless politician; it's that there is disturbing evidence that the same sentiment may be taking hold among those who set the direction of scientific research in the US.

*Correspondence: petsko@brandeis.edu

Rosenstiel Basic Medical Sciences Research Center, Brandeis University, Waltham, MA 02454-9110, USA
I know what you're probably thinking: "Wait a minute. I thought the direction of scientific research in the US was set by peer review of research applications. Are you saying that research scientists are clueless about the importance of the fruit fly as a model organism?"

No, I'm not. I think most practicing biologists understand very well the vital role that model organism research has had in the development of their field, and should continue to have in the future. But if you think that the direction of scientific research is still set by open competition in grant review panels, you should think again.

One of the most disturbing recent trends in the politics of scientific funding has been the creeping hegemony of top-down prioritization. When Vannevar Bush created the modern edifice of government-supported basic research in the 1950s (before that time science was largely supported by small grants from one's home institution or funds from industry), he envisioned that the direction of such research would be set by curiositydriven, investigator-initiated research proposals competing for funding in open peer-review panels. And for more than 40 years, with occasional hiccups (for example, President Nixon's War on Cancer), that was the case. I think most objective observers would concur that, as an engine for driving innovation, the system worked amazingly well - especially compared with more topdown, managed science structures such as those in Japan and many European countries.

But then two things happened. One was the increasing clamor by patient advocacy groups for biomedical delivery on the promises that had been made to justify the big increases in funding during the previous decades. Now, I happen to believe that there have actually been far more successes than the public are aware of - we simply have done a terrible job of getting those stories across. But this is clearly one case where perception trumps reality. And as science administrators came under fire by impatient groups of patients, they naturally responded by trying to take more control of the scientific enterprise into their own hands, so it could be steered towards more direct pay-offs.

The other disruptive event was the success of the human genome project. By 'success' I don't mean scientifically - though it certainly was successful that way. 
I mean its success in making anyone connected with it the scientists who led it, and the bureaucrats who supported it - famous and in advancing their careers. Plus, you will recall that the human genome project was initiated by a small group of scientists against the objection of many of their colleagues that it would divert funds away from small basic research projects. The lessons that were learned was that it paid to set the direction of research from the top down and that big science programs were a rising tide that could lift, if not all boats, then certainly the boats that were tied to them.

Naturally, the human genome project led to other human fill-in-the-blanks-omics projects. Equally naturally, these were paraded before the public as examples of how the biomedical research establishment was trying to satisfy their demand for results. And so, inexorably over the past 15 years, it has become easier to get research that is closely related to human biology and human disease funded than research that is more distantly related.

Among the casualties of this trend is model organism research, and not just in the halls of the funding agencies. One of the outstanding examples of the success of model organism approaches in recent years has been the work on models for neurodegenerative diseases. The difficulties in working directly with the human central nervous system vanish when one uses flies or worms or fish; and the intracellular processes involved in neurodegeneration can be modeled down to some extent down to unicellular eukaryotes like yeast. Drug screens in yeast and screens for disease modifier genes in yeast, worms, flies, and fish have yielded results that translate cleanly to disease models in mice and rats. There are potential therapeutics entering animal toxicity studies, prior to human clinical trials, for devastating disorders like Parkinson's disease that were first identified from screens in much lower organisms. And most of the fundamental intracellular pathways that are involved in numerous human diseases were first identified in such organisms and continue to be identified first in such models - a recent example being autophagy, which was discovered and characterized in yeast.

Despite numerous examples of the enormous value of model organism research, many scientists and scientific administrators seem to think that nothing lower than a mouse is suitable for cutting-edge biology anymore. I have seen reviews of submitted papers in which the referee clearly does not believe it is possible to gain insight into human diseases from studies in yeast models never mind more than 20 years of evidence to the contrary. Grant applications to support such research also have a similar gauntlet to run.

The current love affair with mouse models is particularly troubling to me because it's not clear that mice are all that good as a model for many human diseases. Why would one even expect that a relatively genetically homogeneous mouse population, raised in a sterile environment, recapitulates salient features of the mongrel-like, flora- and fauna-infested human race? An alarmingly high percentage of genes produce no phenotype when they are knocked out in a mouse. Mouse models for neurodegenerative diseases are notoriously bad: in almost no case is the full range of human pathology, including histopathology, faithfully reproduced (perhaps because mice simply don't live long enough), so it's far from certain that one learns as much from them as from studies in simpler, less-expensive organisms for which a wider range of techniques are available.

Plus, there is the fact that human disease models are suspiciously easy to cure in mice. Most non-biological drugs that are taken into human clinical trials fail not in Phase 1, where toxicity is assessed, but in Phase 2, where efficacy is evaluated, suggesting that mouse models for many human diseases, which are the most common ones used to evaluate efficacy in pre-clinical trials, are woefully inadequate. (There is some hope that rat models may be better for some neurological disorders, but it's too early to tell yet.)

Basic research discoveries, which are the wellspring of major advances in medicine and other applied areas of science, are likely to come from research in simple systems for the foreseeable future, which makes the rush to displace them with mammalian models hard to justify. (I'm not trying to trash all mammalian models here; one will always have need for both.) We need to keep reminding the powers that be, who seem hell-bent on setting the direction of research in the US, that fundamental work in lower organisms remains incredibly important.

If you want an example to show them, a recent issue of Nature provides a good one. The cover articles are reports of two breakthrough discoveries in melanoma - a deadly and hard-to-treat skin cancer - that were made by studying zebrafish. It has been known for some time that the most common mutation in human melanoma is $B R A F(V 600 E)$, which activates the oncogenic protein kinase BRAF; however, such mutations also occur in benign lesions, highlighting the importance of multiple causative loci, as Hannahan and Weinberg pointed many years ago out must be true for most, if not all, cancers. To pinpoint one such mutation, Ceol et al. (Nature 471:513$517,2011)$ developed an assay to test candidate genes in transgenic zebrafish expressing BRAF(V600E), the equivalent of a classic synthetic lethal screen in genetics. The study identified SETDB1, which encodes a histone methyltransferase, as the gene harboring the second genetic lesion (an amplification). Further analysis showed that increased levels of SETDB1 dysregulated a number 
of important genes, leading to accelerated onset of melanoma. In the second report, White et al. (Nature 471:518-522, 2011) used zebrafish embryos to identify the transcriptional events that occur when BRAF is activated. Because melanocytes derive from the neural crest during development, a chemical genetics screen was performed for compounds that would suppress the neural crest lineage during zebrafish development; any hits were then tested for their effects on melanoma models. Inhibitors of the enzyme dihydrooritate dehydrogenase led to an almost complete abrogation of neural crest development in the fish, and to a reduction in selfrenewal of mammalian neural crest stem cells. These same inhibitors markedly decreased melanoma growth in vitro and in mouse xenograft models.

It may be that both of these studies could have been carried out with equal success in human cell culture or mouse studies, but they weren't, and it's pretty certain that they could not have been carried out as efficiently, if at all. The progression from the model organism studies in fish, which identified novel pathways and targets, to the follow-up work in mammals and mammalian cells can be extremely powerful, as shown here, but will it be possible to obtain support for such a progression in the future if only work in higher organisms is deemed relevant to human disease?

Obviously, things have not gone so far - yet - that research on model organisms is impossible to get funded or published. But I don't like the trend, and I think it will take constantly reminding our administrators - and our peers - of the value of such research to combat the encroaching belief, which is probably not even true for behavior, that the proper study of mankind is man - or at least mice and men.

If science administrators adopt the dogma that human biology can be uncovered only by studies on mammalian cells or on organisms at least as complex as a mouse; if the people who allocate research funds are convinced that human diseases cannot be modeled effectively in less complex, but more genetically and biochemically tractable, organisms; if scientists forget the grand unity of biology that underlies all of the great advances that have been made by studying simple systems ranging from bacteriophage to zebrafish; if we all succumb, in other words, to a Sarah Palin-esque view of the world, then our ability to innovate will slow to a worm-like crawl; the forces of ignorance will continue to rise like yeast; we will be swimming upstream in our attempt to bring the fruits of genomics to the public; and projects that have everything to do with the public good will die like flies. I kid you not.

Published: 30 May 2011

doi:10.1186/gb-2011-12-5-115

Cite this article as: Petsko GA: In praise of model organisms. Genome Biology 2011, 12:115. 